\title{
Dipolar oscillations in a quantum degenerate Fermi-Bose atomic mixture
}

\author{
F. Ferlaino, R. J. Brecha $\dagger$ P. Hannaford $\ddagger$ F. Riboli, G. Roati, \\ G. Modugno, M. Inguscio \\ European Laboratory for Nonlinear Spectroscopy, Universitá di Firenze, and Istituto \\ Nazionale per la Fisica della Materia, Via Nello Carrara 1, 50019 Sesto Fiorentino, \\ Italy. \\ † Physics Department, University of Dayton, Dayton, OH 45469-2314 USA. \\ $\ddagger$ Swinburne University of Technology, Mail H31, PO Box 218, Melbourne, Australia.
}

\begin{abstract}
We study the dynamics of coupled dipolar oscillations in a Fermi-Bose mixture of ${ }^{40} \mathrm{~K}$ and ${ }^{87} \mathrm{Rb}$ atoms. This low-energy collective mode is strongly affected by the interspecies interactions. Measurements are performed in the dynamical and quantum degenerate regimes and evidence the crucial role of the statistical properties of the mixture. At the onset of quantum degeneracy, we investigate the role of Pauli blocking and superfluidity for $\mathrm{K}$ and $\mathrm{Rb}$ atoms, respectively, resulting in a change in the collisional interactions.

PACS numbers: 05.30.Jp, 05.30.Fk,34.50.-s, 34.50.Pi
\end{abstract}

Submitted to: J. Opt. B: Quantum Semiclass. Opt. 


\section{Introduction}

Recent research on ultracold atomic mixtures has opened a new scenario in the field of quantum degenerate gases. Thanks to sympathetic cooling techniques, it is now possible to study a variety of degenerate mixtures composed of two bosonic species [1, 2] as well of bosonic and fermionic isotopes of the same species [3, 4] or of different species [5, 6]. In a mixture, the interactions between unlike particles determine relevant properties such as the efficiency of rethermalization processes or the stability of the mixture against collapse [7, 8]. They also could play a crucial role in the study of atoms-to-molecule association [9] and in the binding energy for BCS pairing of fermions [10]. The elastic interactions between ultracold gases can be investigated by the study of the damping of dipolar oscillations. This method was originally used in [11] for a two components BoseEinstein condensate (BEC) loaded in different hyperfine levels and was then extended to a gas of fermions in two spin-states [12 and to a mixture composed of different atoms [13]. In a harmonic potential, single gases undergo undamped collective oscillations, whereas two gases experiencing different confinements can exhibit a damped out-ofphase motion giving quantitative information about the scattering processes. In the degenerate regime, the dipolar oscillations are affected by the quantum statistic and their study can give useful information on the mean-field interactions between the two gases.

In the present work we apply for the first time this technique to a degenerate Fermi-Bose mixture. The study of ${ }^{40} \mathrm{~K}-{ }^{87} \mathrm{Rb}$ dipolar oscillations gives a signature of the strong interspecies interactions. We investigate how the damping is modified when one or both gases are brought to the quantum degenerate regime. We observe a transition from hydrodynamic to collisionless regime which reveals the roles of Pauli blocking of

collisions at the onset of Fermi degeneracy. At lower temperatures, when also BEC occurs, we evidence also the effect of superfluidity of bosons on the damping.

\section{Experimental}

The ultracold $\mathrm{K}-\mathrm{Rb}$ mixture is produced by a combination of magneto-optical trapping and sympathetic cooling in a magnetic trap. The experimental apparatus and techniques are described in detail in Refs. [1, 6]. In brief, about $10^{9} \mathrm{Rb}$ atoms and $10^{5} \mathrm{~K}$ atoms at a temperature $T=100 \mu \mathrm{K}$ are loaded in a quadrupole-Ioffe configuration (QUIC) magneto-static trap. Here Rb-selective evaporative cooling is performed using radio-frequency radiation, taking advantage of the different Zeeman structure of the two species. Potassium atoms are sympathetically cooled through elastic interspecies collisions. With an evaporation ramp lasting about $25 \mathrm{~s}$ we are able to cool typically a few $10^{4}$ atoms to the quantum degenerate regime. For these numbers of atoms, the Fermi temperature for $\mathrm{K}$ is of the order of $T_{F}=300 \mathrm{nK}$ and the critical temperature for BEC of $\mathrm{Rb}$ is $T_{c}=150 \mathrm{nK}$. In these conditions, the BEC is usually completely surrounded by the Fermi gas, whose dimensions are approximatively as twice as large [6]. Both species 
are trapped in their stretched spin states, $\left|F=9 / 2, m_{F}=9 / 2\right\rangle$ for $\mathrm{K}$ and $|2,2\rangle$ for $\mathrm{Rb}$. These states experience the same trapping potential, with axial and radial harmonic frequencies $\omega_{a}=2 \pi \times 24 \mathrm{~s}^{-1}$ and $\omega_{r}=2 \pi \times 317 \mathrm{~s}^{-1}$ for $\mathrm{K}$, while those for Rb are a factor $\left(M_{R b} / M_{K}\right)^{1 / 2} \approx 1.47$ smaller. The different trapping frequencies experienced by the two gases allows one to induce a relative motion between the two components.

To excite dipolar oscillations of the two samples we suddenly displace the minimum of the trapping potential in the horizontal direction $x$, by changing the ratio of currents in the trap coils. By an appropriate choice of the amplitude and timing of such displacement we can excite a quasi-pure dipolar oscillation, with no apparent higherorder (shape) oscillations. The typical mean relative velocity of $\mathrm{K}$ and $\mathrm{Rb}$ samples during the subsequent oscillations is $\sqrt{\left\langle v^{2}\right\rangle}=5 \mu \mathrm{m} / \mathrm{ms}$. We note that the amplitude of oscillations is small enough to preserve the overlap of the two clouds even in the degenerate regime [6].

The centers-of-mass positions of both samples in the magnetic trap can be determined simultaneously at the end of each experimental run by means of two-color absorption imaging. For this, we use two delayed light pulses, at $767 \mathrm{~nm}$ for $\mathrm{K}$ and $780 \mathrm{~nm}$ for Rb, lasting $30 \mu \mathrm{s}$ each, which are imaged on different areas of a CCD sensor. The atoms are imaged after a ballistic expansion following release from the trap. The expansion times for $\mathrm{K}$ and $\mathrm{Rb}$ are $4 \mathrm{~ms}$ and $19 \mathrm{~ms}$ respectively.

\subsection{Thermal gases: K-Rb scattering length}

In a first set of measurements we have studied the damping of the dipolar oscillations for a nondegenerate $\mathrm{K}-\mathrm{Rb}$ sample, at temperatures $T=300-500 \mathrm{nK}$. The effect of the interspecies collisions is apparent from the data in Fig. 1: both $\mathrm{K}$ and Rb oscillations are damped, and the $\mathrm{K}$ motion is also frequency-shifted.

To describe the coupled center-of-mass motion, we use a classical model for two harmonic oscillators coupled through a viscous damping. The coupled equations of motion are

$$
\begin{aligned}
& \ddot{x}_{R b}=-\omega_{R b}^{2} x_{R b}-\frac{4}{3} \frac{M_{K}}{M} \frac{N_{K}}{N} \Gamma_{\text {coll }}\left(\dot{x}_{R b}-\dot{x}_{K}\right) \\
& \ddot{x}_{K}=-\omega_{K}^{2} x_{K}+\frac{4}{3} \frac{M_{R b}}{M} \frac{N_{R b}}{N} \Gamma_{\text {coll }}\left(\dot{x}_{R b}-\dot{x}_{K}\right),
\end{aligned}
$$

where $M$ is the total mass $M_{K}+M_{R b}, N$ the total number of atoms $N_{K}+N_{R b}$, and $\Gamma_{\text {coll }}$ is the rate of $\mathrm{K}-\mathrm{Rb}$ two-body elastic collisions. This model can be used to describe the center-of-mass motion of the two clouds, whereas all the microscopic damping mechanisms are described by the quantity $\Gamma_{\text {coll }}$. Assuming two Boltzmann distributions for the gases at sufficiently low temperature $T$ to be in the Wigner regime, one obtains

$$
\Gamma_{\text {coll }}=\bar{n} \sigma v_{t h},
$$

where $v_{t h}=\sqrt{8 k_{B} T / \pi M}$ is the rms relative thermal velocity and $\bar{n}=\left(\frac{1}{N_{K}}+\right.$ $\left.\frac{1}{N_{R b}}\right) \int n_{K} n_{R b} d^{3} x$. Finally, the collision cross-section $\sigma$ depends on the interspecies s- 


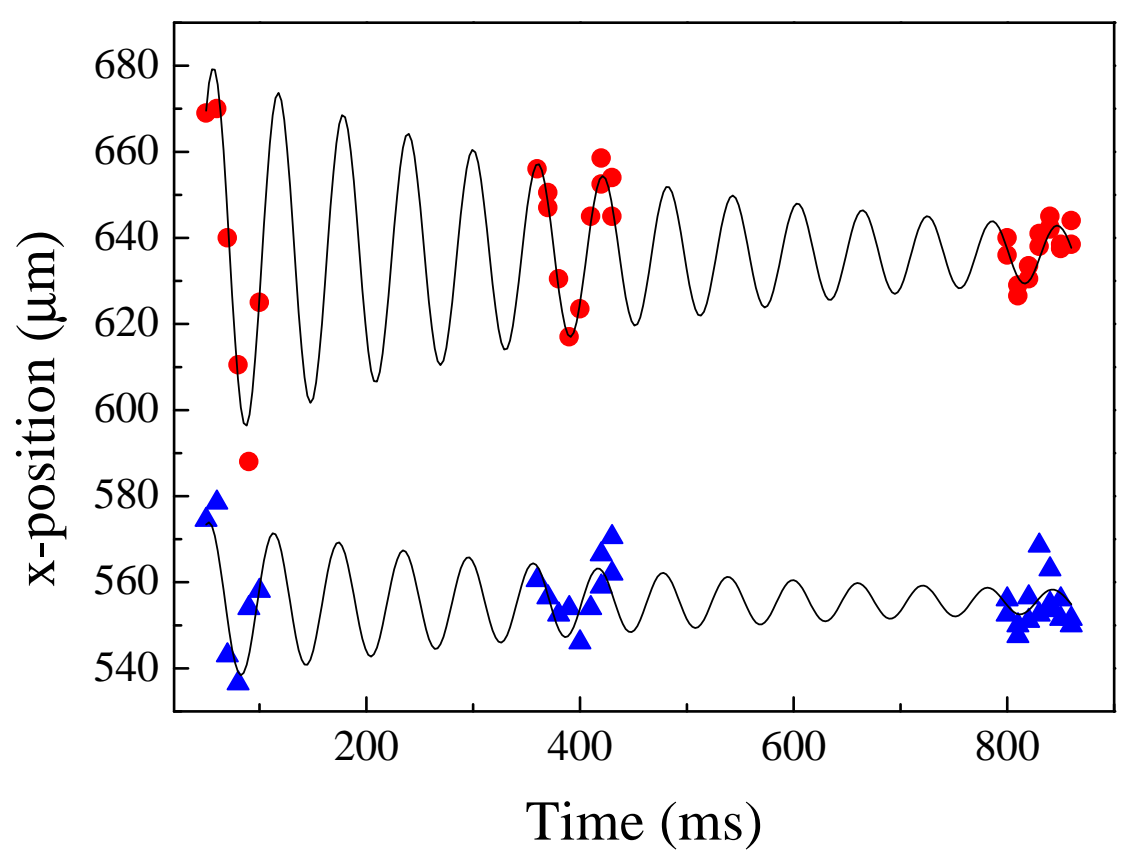

Figure 1. Coupled dipolar oscillations of $8 \times 10^{3}$ nondegenerate $\mathrm{K}$ (triangles) and $8 \times 10^{4}$ uncondensed $\mathrm{Rb}$ (circles) atoms along the horizontal axis at $T=300 \mathrm{nK}$. The two samples oscillate at the same frequency with a collisional rate $\Gamma_{\text {coll }}=190 \mathrm{~s}^{-1}$ typical of a hydrodynamic regime. The solid lines are the best fit to the model presented in the text.

wave scattering length as

$$
\sigma=4 \pi a^{2}
$$

The motion of two coupled harmonic oscillators is a well know problem, and here we just recall the properties which are important for the present experiment. In general such a system has two normal modes, whose frequencies $\omega$ and damping time $\tau$ vary with the collisional rate $\Gamma_{\text {coll }}$. The behaviour, calculated by numerically solving Eq. 1 for the ratio of atom numbers that we have typically in the experiment, $r=N_{R b} / N_{K}=7.5$, is shown in Fig. 2. At low collisional rate, in the so-called collisionless regime $\left(\omega_{R b} \tau, \omega_{K} \tau \ll 1\right)$, the two samples are predicted to oscillate at their bare frequencies $\left(\omega_{K} \approx 2 \pi \times 24 \mathrm{~s}^{-1}\right.$, $\omega_{R b} \approx 2 \pi \times 16.3 \mathrm{~s}^{-1}$ ), and the ratio of the two damping times scale as the inverse ratio of the total mass of each sample. As the collisional rate increases, the damping time of the two normal modes decreases and their frequencies are shifted towards an intermediate value. Here each sample oscillates at a combination of both normal modes. Finally, at very high collisional rate $(\omega \tau>1)$ the system enters the hydrodynamic regime. Here there is a mode at this intermediate frequency with low damping and a second overdamped mode whose frequency rapidly decreases with increasing $\Gamma_{\text {coll }}$. This situation corresponds to what we observe in the experiment ( see Fig. 1), where the two 
samples oscillate at the same frequency, almost in phase and with a long damping time. In Fig. 2 we also report the measured damping time vs the collisional rate, that confirm the prediction of an increasing $\tau$ for increasing $\Gamma_{\text {coll }}$. For example in Fig. 3 we show the oscillations of a sample composed of three times as many atoms of each species as that in Fig. 1. As predicted, the damping time is three times longer than the one observed in Fig. 1.

The dependence of $\tau$ on $\Gamma_{\text {coll }}$ in the hydrodynamic regime is weaker than in the collisionless regime (see Fig. 2b), while instead there is a strong dependence of the relative phase of $\mathrm{K}$ and $\mathrm{Rb}$ motion on $\Gamma_{\text {coll }}$. The latter dependence helps to provide an accurate determination of the experimental $\Gamma_{\text {coll }}$, since the phase of the oscillations can be determined with relatively high accuracy.

The overall behaviour of the normal modes of the coupled oscillators can be qualitatively understood by noting that an increasing collisional rate tends to restore local equilibrium in the mixture. For the system, the way to produce this with the lowest energy cost is to shift the oscillation frequency of the sample with the smaller mass, and then to reduce the phase difference between the two samples. These features do not change significantly when changing the number ratio $r$. In particular, we find that the minimum damping rate at the transition from the collisionless to the hydrodynamic regime is always around $140 \mathrm{~s}^{-1}$, whereas the region where the frequencies of the two modes cross is centered around $220 \mathrm{~s}^{-1}$, irrespective of $r$. In any case, we note that this crossing occurs only for $r>1$; for smaller ratios it is the "potassium mode", i.e. the high frequency mode, that acquires long damping times in the hydrodynamic regime.

The experimental values of the collisional rate can be used to determine the K-Rb s-wave triplet scattering length. To obtain the value that we have already reported in [8], we fit the experimental data for the dipole oscillations with the solution of Eq. [1, and we extract a value for the collision cross-section and hence for the scattering length $a$. We have repeated this procedure by varying the temperature in the range $T=300$ $500 \mathrm{nK}$, the total number of atoms in the range $N=10^{4}-5 \times 10^{5}$, and the ratio $r$ from 2.5 to 7.5. We have then made a weighted average of the resulting values for the scattering length and obtained $|a|=410_{-80}^{+90} a_{0}$. Here the uncertainty is dominated by a $40 \%$ a priori uncertainty in the number density.

\subsection{Fermi gas and thermal bosons: Pauli blocking of collisions}

We have repeated the above experiments at lower temperatures, with one or both gases in the degenerate regime. As we will show in the following, the study of dipolar oscillations in this regime can reveal effects caused by the modification of the collisional properties of a degenerate gas. The quantitative analysis of experimental results is, however, complicated by the fact that at finite $T$ the bosonic sample has both a condensed and an uncondensed fraction, which are expected to interact in different ways with fermions. To simplify the analysis we have first investigated the interaction between the degenerate Fermi gas and an uncondensed bosonic cloud. 


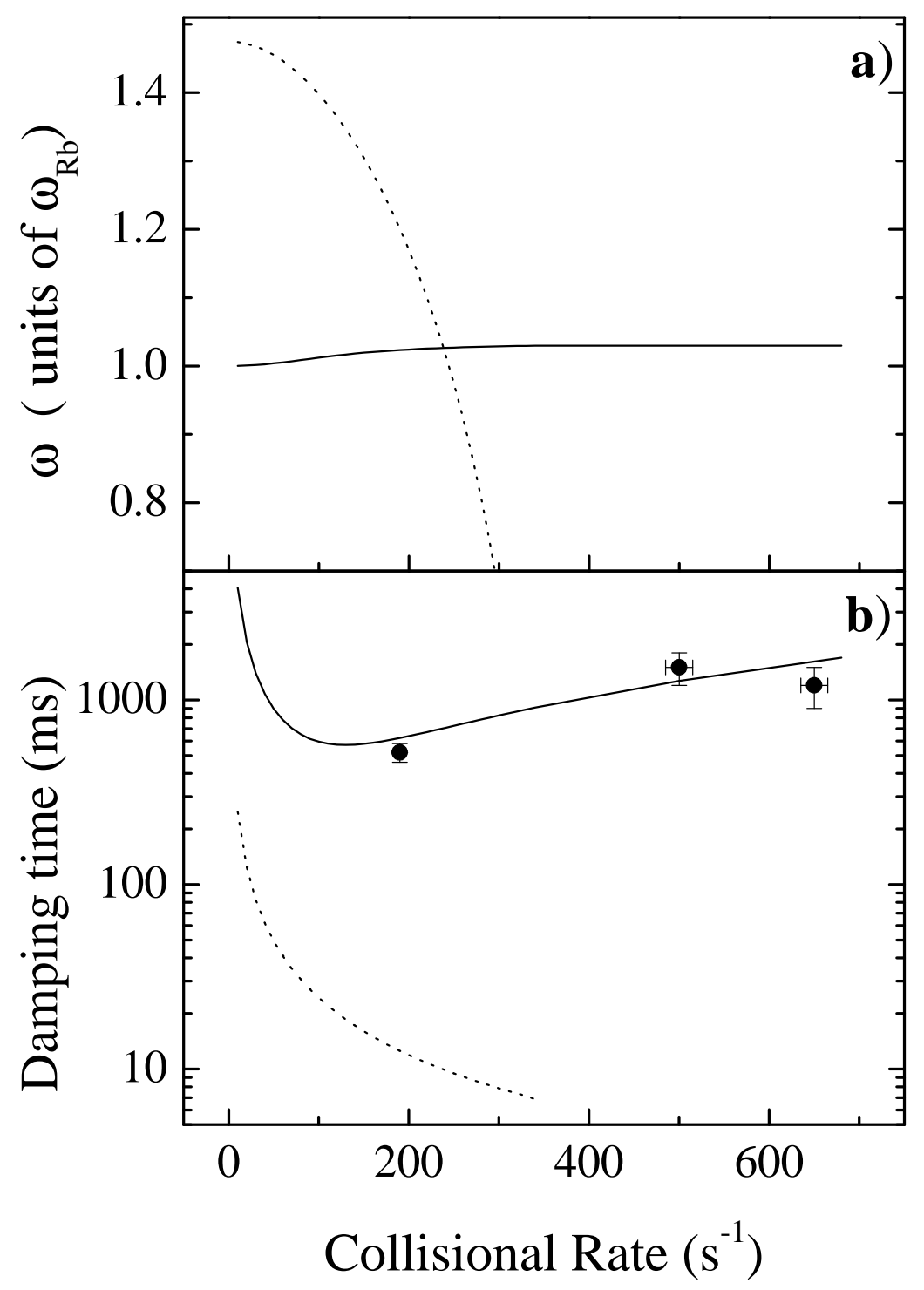

Figure 2. Oscillation frequency (a) and damping time (b) for the two normal modes of the K-Rb coupled dipole oscillations, as a function of the collisional rate. The curves are calculated according to the classical kinetic model (see Eq. 1), for a sample composed of $\sim 88 \%$ of $\mathrm{Rb}$ (solid line) and $\sim 12 \%$ of $\mathrm{K}$ (dashed line), with an interspecies scattering length $a=-410 a_{0}$. The points in (b) are the experimental data for a thermal sample in the hydrodynamic regime. Here we are not able to observe the strongly damped out-of-phase motion.

In the experiment, this situation is actually easier to produce than the opposite case. Indeed, for the same number of atoms of each species, the Fermi temperature is higher than the critical temperature for condensation also because of the different 


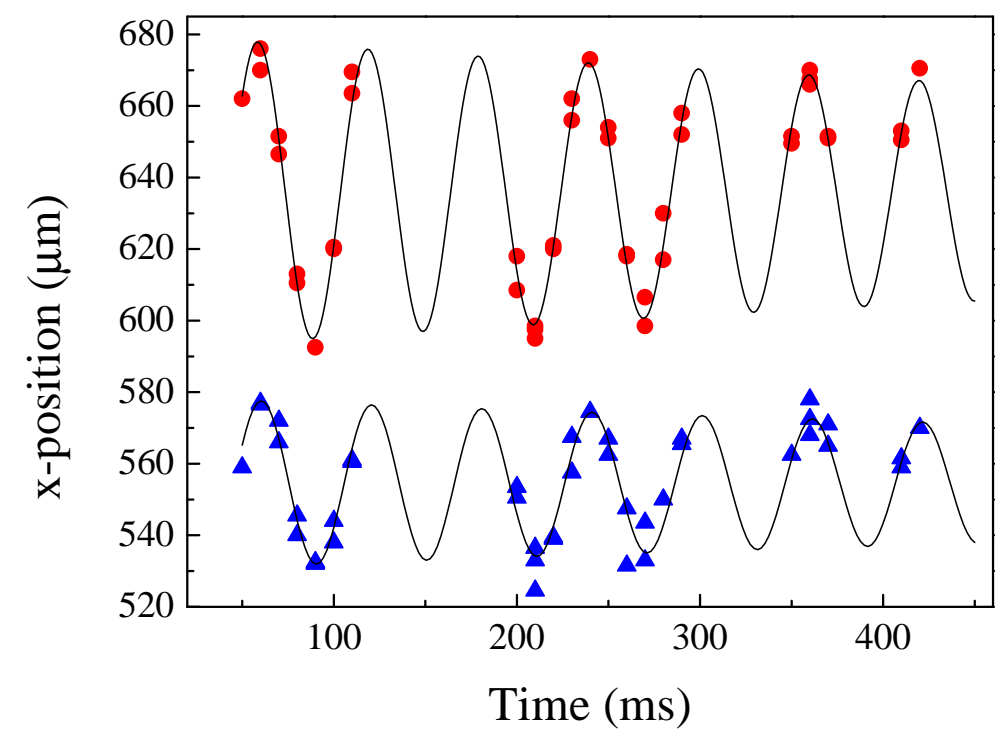

Figure 3. Coupled dipolar oscillations of $2 \times 10^{4}$ thermal K (triangles) and $2 \times 10^{5} \mathrm{Rb}$ (circles) atoms along the horizontal axis in the hydrodynamic regime at $T=300 \mathrm{nK}$. The two samples oscillate at the same frequency with a collisional rate $\Gamma_{\text {coll }}=650 \mathrm{~s}^{-1}$. The solid lines are the best fit to the model presented in the text (see Eq. 1).

confinements:

$$
\begin{aligned}
& k_{B} T_{F}=\hbar \bar{\omega}_{K}\left(6 N_{K}\right)^{1 / 3}, \\
& k_{B} T_{c}=\hbar \bar{\omega}_{R b}\left(N_{R b} / 1.26\right)^{1 / 3} .
\end{aligned}
$$

To produce the mixed sample we intentionally force the final stage of evaporation of $\mathrm{Rb}$, in order to reduce the atom number and prevent the onset of BEC. Using this procedure we have been able to produce a sample composed of $2 \times 10^{4} \mathrm{~K}$ atoms and $10^{4}$ Rb atoms at $T=120 \mathrm{nK}$, for which $T_{F}=260 \mathrm{nK}$ and $T_{c}=110 \mathrm{nK}$. In this condition, we can calculate the damping rate for two classical samples $\Gamma_{\text {coll }}=100 \mathrm{~s}^{-1}$ which correspond to a mixture in the hydrodynamic regime.

In Fig. . 1 we show the evolution of the coupled dipolar oscillation for this particular sample. Note that in this case each of the two species oscillates at its bare frequency $\left(\omega_{K} \approx 2 \pi \times 24 \mathrm{~s}^{-1}, \omega_{R b} \approx 2 \pi \times 16.3 \mathrm{~s}^{-1}\right)$; this indicates that the collisional rate is now smaller than the oscillation frequencies, i.e. we are in the collisionless regime. From a fit we obtain $\Gamma_{\text {coll }}=60(10) \mathrm{s}^{-1}$, which is significantly smaller than the one expected for a nondegenerate sample. This is a clear indication that the interspecies collisional processes are somewhat reduced once the Fermi gas is cooled below $T_{F}$. This phenomenon is actually predicted to occur because of the Pauli blocking of elastic collisions [14: at $T \ll T_{F}$ only the fermions in the outer shell of the Fermi sphere, having an energy $E=E_{F}-E_{C M}$ can scatter with bosons and participate in the damping. Here $E_{C M}$ is the 


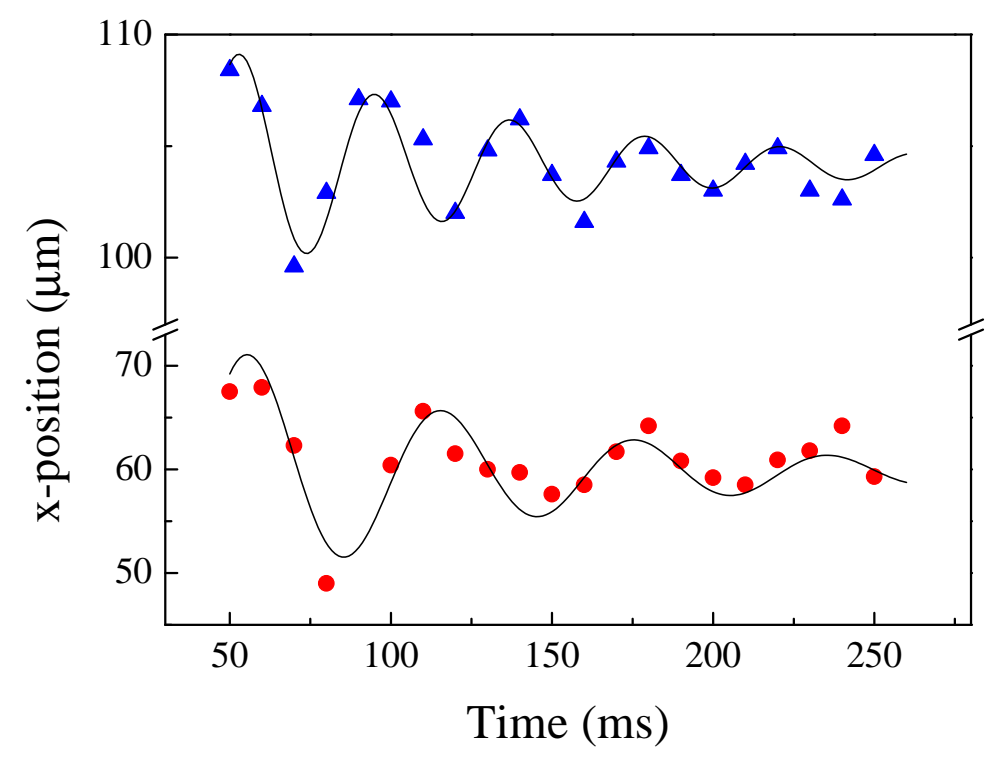

Figure 4. Coupled dipolar oscillations of degenerate $\mathrm{K}$ (triangles) and thermal $\mathrm{Rb}$ (circles) clouds along the horizontal axis in the collisionless regime at $T=120 \mathrm{nK}$. The two samples oscillate at their bare frequencies with a collisional rate $\Gamma_{\text {coll }}=60 \mathrm{~s}^{-1}$ typical of a collisionless regime. The solid lines are the best fit to the model presented in the text.

collision energy of fermions and bosons in the center-of-mass frame

$$
E_{C M}=\mu\left\langle v^{2}\right\rangle / 2,
$$

where $\mu$ is the reduced $\mathrm{K}$-Rb mass. An upper value for $E_{C M}$ for the typical experimental parameters is $E_{C M} / K_{B} \approx 50 \mathrm{nK}$, which is significantly smaller than the typical Fermi energy $E_{F} / K_{B}=300 \mathrm{nK}$. At $T=0 \mathrm{~K}$, just half of the fermions can participate to the scattering processes with bosons, however a finite temperature reduces Pauli blocking because of the smearing of the Fermi distribution. As shown in [15], for this excitation energy Pauli blocking should nevertheless produce a significant reduction of $\Gamma_{\text {coll }}$, also at the temperature $T=0.4 T_{F}$ of the experiment, as indeed we observe.

The Pauli blocking phenomenon can be viewed as a tool to investigate the level of degeneracy of a trapped fermionic gas. In a degenerate Fermi gas, in contrast to a thermal cloud, the spatial density and the mean speed of the atoms remain constant when lowering the temperature. This deviation from classical behaviour does not allow direct measurement of the sample temperature. Since the reduction of the damping rate is predicted to depend on $T$, the damping of relative motion could be used to determine the temperature of fermions even in the regime where boson thermometry does not work anymore, as suggested in [15] and in [16]. A quantitative investigation of the phenomenon needs to be supported by an appropriate quantum model for the collisions. 


\subsection{Fermi gas and BEC: superfluidity}

Finally, we have repeated the experiments in the quantum regime using a degenerate Fermi gas and a BEC. We observe a crossover from the hydrodynamic to the collisionless regime when the temperature of the bosons is lowered by evaporation below $T_{c}$. In Fig. 5a, we show strongly damped Rb and K oscillations in the crossover region between the hydrodynamic and collisionless regimes. Here the motion of the two clouds is the superposition of two damped oscillations at frequencies close to the bare frequencies of the two clouds. Here the dipole oscillations are excited for a mixed sample with $N_{K}=2.5 \times 10^{4} \mathrm{~K}$ atoms and $N_{R b}=3.9 \times 10^{4}$ at $T=0.84 T_{c}=118 \mathrm{nK}$. In Fig. $5 \mathrm{~b}$ we show a measurement performed with a BEC at even lower a temperature $T<0.7 T_{c}$. Here the two samples oscillate at their bare frequencies, indicating that we are in the collisionless regime. Notice that the minimum measurable temperature is limited to $T=0.7 T_{c}$ by the minimum detectable uncondensed $\mathrm{Rb}$ component. .

The variation of the collisional rate as a function of the reduced boson temperature is shown in Fig. 6, for different mixed samples in a typical evaporation across the phasetransition to BEC. The number of Rb atoms varies from about $2 \times 10^{5}$ at the highest temperature to $10^{4}$ at the lowest temperature because of losses due to evaporation, whereas the number of $\mathrm{K}$ atoms stays almost constant $\left(N_{K} \approx 2 \times 10^{4}\right.$, corresponding to a Fermi temperature $T_{F} \approx 2 T_{c}$ ). To understand the role of the varying temperature and atom number on the measured collisional rate, we have computed for each experimental point the corresponding expected rate for a classical gas (circles in Fig. 6). This collisional rate is calculated from Eq. 2, assuming that both $\mathrm{K}$ and $\mathrm{Rb}$ atoms follow a Boltzmann distribution at all temperatures. The classical prediction is clearly larger than the observed $\Gamma_{\text {coll }}$ for $T<T_{c}$, indicating that quantum degeneracy of the mixed system is influencing the fermion-boson interaction.

Apart from the Pauli blocking already addressed above, one should also consider the superfluid behavior of the BEC [17]. Collisions between the BEC and fermions are indeed suppressed if the momentum exchanged in the collision is below $M_{R b} c$, where $c$ is the

sound velocity $c=\sqrt{4 \pi \hbar^{2} a_{R b} n_{0} / M_{R b}^{2}}$. Note in particular the dramatic reduction of $\Gamma_{\text {coll }}$ when lowering the temperature from $T_{c}$ to $0.85 T_{c}$, which cannot be reproduced by the classical prediction. Since the change in temperature between these two measurements is negligible for fermions, the reduction in collisional rate cannot be ascribed to Pauli blocking in the Fermi gas, and this appears to be the consequence of superfluidity in the BEC.

To gain a more quantitative insight into this phenomenon we study in detail the measurement at $T=0.85 T_{c}$ in which the condensed and uncondensed fractions are detectable. To model the system in these conditions, we modify Eq. 1 to include a third harmonic oscillator, i.e. the uncondensed Rb cloud, which is coupled to both the $\mathrm{BEC}$ and the Fermi gas. In the experiment we were able to follow the center-of-mass motion of both the condensed and uncondensed components of the Rb sample. The two motions occurred at the same frequency and exactly in phase, although they were 


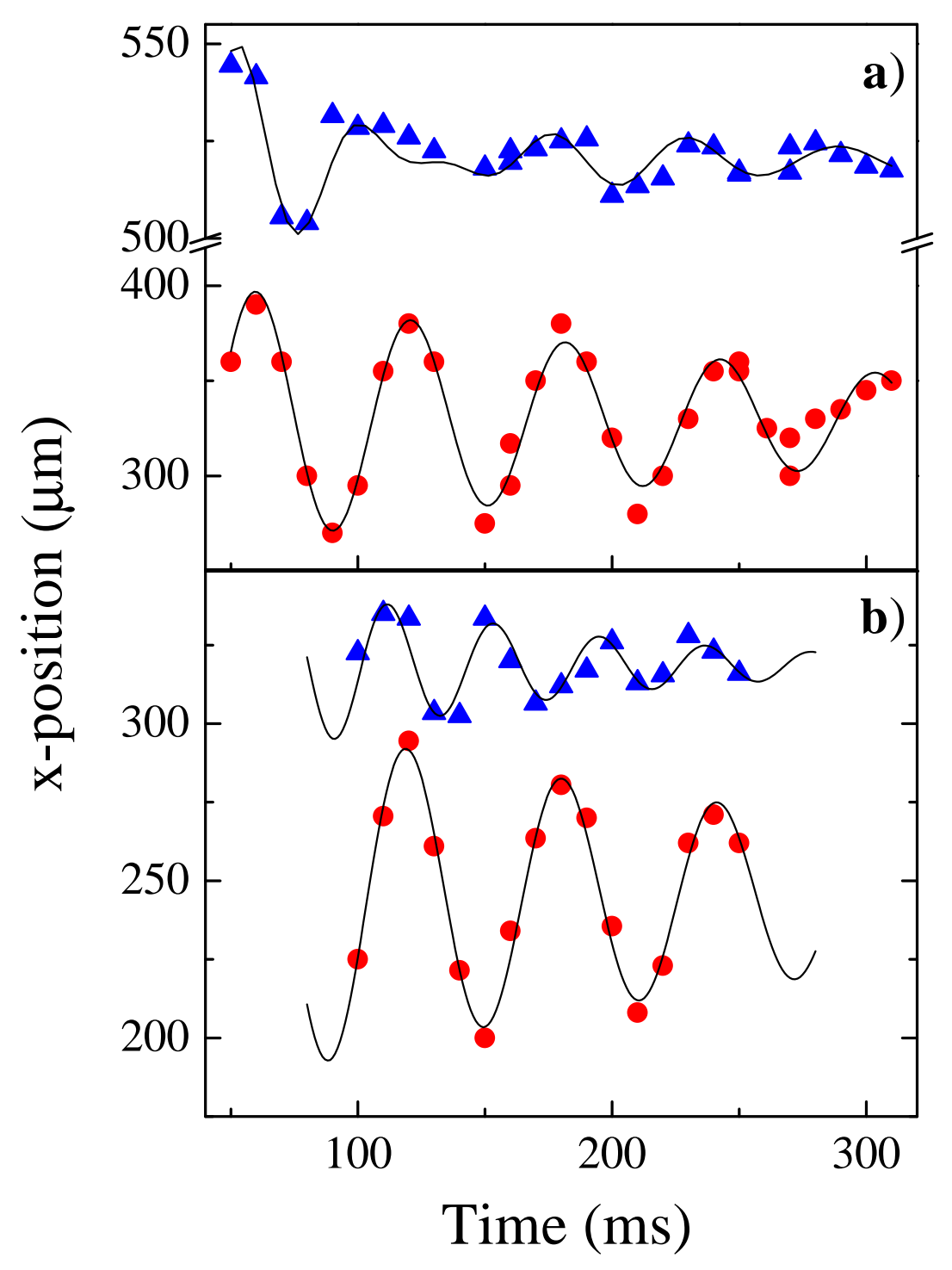

Figure 5. Coupled dipolar oscillations of degenerate $\mathrm{K}$ (triangles) and Rb BEC (circles) clouds along the horizontal axis: (a) in the crossover between Hydrodynamic and collisionless regimes at $T \approx 0.84 T_{c}$ with $\Gamma_{\text {coll }}=35 \mathrm{~s}^{-1}$ and (b) in the collisionless regime at $T<0.7 T_{c}$ with $\Gamma_{\text {coll }}=50 \mathrm{~s}^{-1}$. The solid lines are the best fit to the model presented in the text.

out of phase with the $\mathrm{K}$ motion. Including this observation, we can therefore write a simplified model that takes into account only the relevant coupling terms

$$
\ddot{x}_{R b}=-\omega_{R b}^{2} x_{R b}-\frac{4}{3} \frac{M_{K}}{M} \frac{N_{K}}{N} \Gamma_{\text {coll }}\left(\dot{x}_{R b}-\dot{x}_{K}\right)
$$




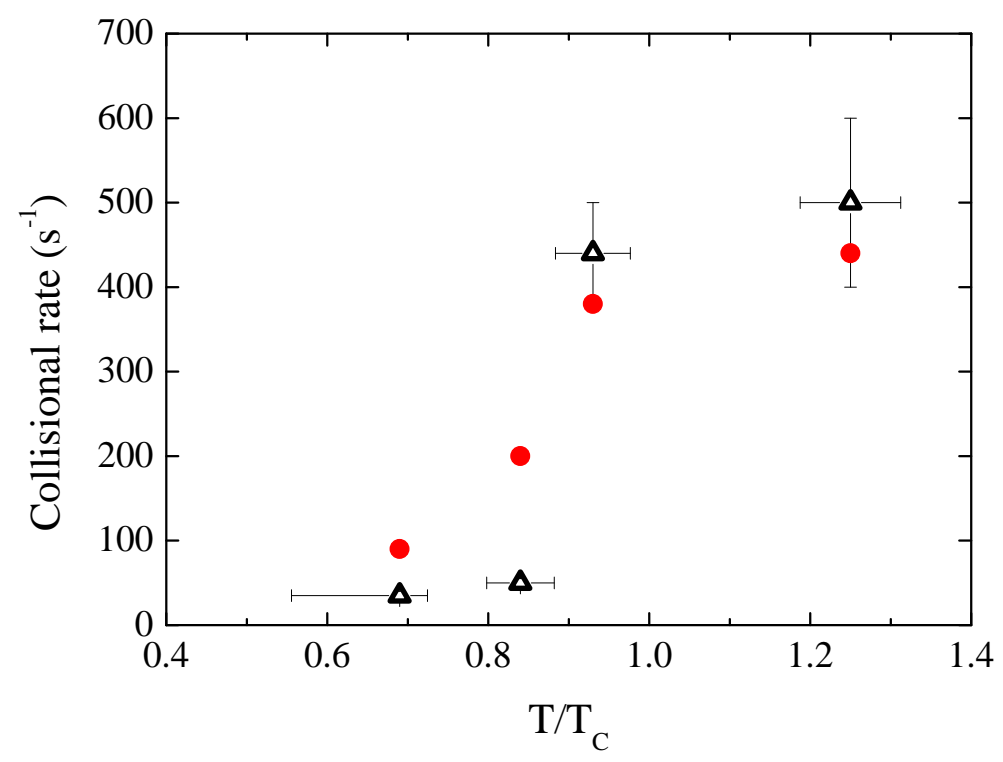

Figure 6. Decrease of the fermion-boson collisional rate as a function of the temperature along a typical evaporation to BEC. For $T<T_{c}$, the experimental rates (triangles) are smaller than the classical prediction (circles) due to the Pauli blocking and superfluidity of $\mathrm{K}$ and $\mathrm{Rb}$ atoms respectively.

$$
\ddot{x}_{K}=-\omega_{K}^{2} x_{K}+\frac{4}{3} \frac{M_{R b}}{M}\left[\frac{N_{R b}}{N} \Gamma_{\text {coll }}+\frac{N_{t h}}{N_{t h}+N_{K}} \Gamma_{t h}\right]\left(\dot{x}_{R b}-\dot{x}_{K}\right),
$$

where $N$ and $M$ are the total number of atoms and mass of the Fermi gas and condensed $\mathrm{Rb}$ fraction, and $N_{t h}$ is the uncondensed $\mathrm{Rb}$ fraction. Here the thermal Rb cloud moves together with the BEC, and interacts with the Fermi gas through the additional damping rate $\Gamma_{t h}$. Since the measurement was performed in almost exactly same conditions as

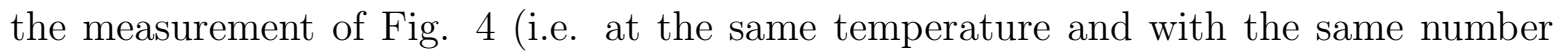
of atoms in the Fermi gas and in the uncondensed Rb cloud), we can fix $\Gamma_{t h}$ to the nominal value determined in that experiment, $\Gamma_{\text {coll }}=60 \mathrm{~s}^{-1}$. Using this value we can unambiguously determine the collisional rate between atoms in the Fermi gas and the condensed fraction of the BEC, $\Gamma_{\text {coll }}=35(10) \mathrm{s}^{-1}$. This value is consistent with the one measured directly in the sample where the uncondensed fraction is not detectable. A damping mechanism that would be taken into account in this condition is the Landau damping. This has been introduced to describe the damping of the relative motion of the BEC condensed and uncondensed components [18, 17, 19]. It could be interesting to extend the study of this mechanism to the Fermi-Bose case. Although the observed damping rates already give evidence of a non-classical behaviour of Fermi-Bose dipolar collisions in the degenerate regime, further modelling is needed to extract quantitative information about the system. One phenomenon that must also be taken into account is the breaking of superfluidity [20], since the relative velocity between the BEC and the Fermi gas is comparable to the sound velocity of our system $c \approx 2 \mu \mathrm{m} / \mathrm{ms}$ during 
collisions.

\section{Discussion and conclusions}

In conclusion we have investigated for the first time coupled dipolar oscillations in a Fermi-Bose dilute gas composed of $\mathrm{K}$ and $\mathrm{Rb}$ atoms. The study of the damping of the oscillations in thermal samples was demonstrated to be a powerful tool for the accurate determination of the interspecies scattering length. The large value of the scattering length in this $\mathrm{K}-\mathrm{Rb}$ pair allowed to access both the collisionless and hydrodynamic collisional regimes by varying the temperature and density of the samples.

At the onset of quantum degeneracy we observe a reduction of the collisional rate. In the interaction of a thermal bosonic gas with the degenerate Fermi gas only the Pauli blocking of collisions is expected to play a role. In the presence of BEC, superfluidity has the effect of lowering the collisional rate. Further modelling of a Fermi-Bose mixture could allow one to extract from the experimental observations useful information on the mean-field interactions [21, 22] and on the temperature of the mixture.

\section{Acknowledgements}

We acknowledge useful discussions with A. Simoni. This work was supported by MIUR, by EC under the Contract HPRICT1999-00111, and by INFM, PRA "Photonmatter" and by the European Science Foundation through the "BEC2000+" programm.

\section{References}

[1] Modugno G, Ferrari G, Roati G, Brecha J R, Simoni A, Inguscio M 2001 Science 294 1320; published online October 18 2001; 10.1126/science.1066687

[2] Modugno G, Modugno M, Riboli F, Roati G, and Inguscio M 2002 Phys. Rev. Lett. 89, 190404

[3] Truscott A G, Streker K E, McAlexander W I, Partridge G B and Hulet R G 2001 Science 291 2570

[4] Schreck F, Khaykovich L, Corwin K L, Ferrari G, Bourdel T, Cubizolles J and Salomon C 2001 Phys. Rev. Lett. 87080403

[5] Hadzibabic Z, Stan C A, Dieckmann K, Gupta S, Zwierlein M W, Gørlitz A and Ketterle W 2002 Phys. Rev. Lett. 88160401

[6] Roati G, Riboli F, Modugno G, and Inguscio M 2002 Phys. Rev. Lett. 89, 150403

[7] Mølmer K, 1998 Phys. Rev. Lett. 80, 1804

[8] Modugno G, Roati G, Riboli F, Ferlaino F, Brecha R J, Inguscio M 2002 Science, 2972240 ; published online August 29 2002, 2001 10.1126/science.1077386

[9] Donley E A, Claussen N R, Thompson S T and Wieman C E 2002 Nature 417, 529

[10] Viverit L 2002 Phys. Rev. A 66, 023605

[11] Maddaloni P, Modugno M, Fort C, Minardi F, and Inguscio M 2000 Phys. Rev. Lett. 85, 2413

[12] Gensemer S D and Jin D S 2001 Phys. Rev. Lett. 87173201

[13] Ferrari G, Inguscio M, Jastrzebski W, Modugno G, Roati G, Simoni A 2002 Phys. Rev. Lett. 89 053202

[14] DeMarco B, Papp S B and Jin D S 2001 Phys. Rev. Lett. 865409

[15] Ferrari G 1999 Phys. Rev. A 59 R4125 
[16] DeMarco B and Jin D S 2002 Phys. Rev. Lett. 88040405

[17] Stamper-Kurn D M, Miesner H J, Inouye S, Andrews M R, and Ketterle W, 1998 Phys. Rev. Lett. $\mathbf{8 1}, 500$

[18] Pitaevskii L P and Stringari S , 1997 Phys. Rev. A 235, 398

[19] Ferlaino F, Maddaloni P, Burger S, Cataliotti F S, Fort C, Modugno M and Inguscio M 2002 Phys. Rev. A $66011604(\mathrm{R})$

[20] Raman C, Kohl M, Onofrio R, Durfee D S, Kuklewicz C E, Hadzibabic Z and Ketterle W 1999 Phys. Rev. Lett. 83, 2502

[21] Miyakawa T, Suzuki T, and Yabu H 2000 Phys. Rev. A 62063613

[22] Sogo T, Miyakawa T, Suzuki T, and Yabu H 2002 Phys. Rev. A 63013618 